\title{
Optical 8-Channel, 10 Gb/s MT Pluggable Connector Alignment Technology for Precision Coupling of Laser and Photodiode Arrays to Polymer Waveguide Arrays for Optical Board-to-Board Interconnects
}

\author{
Ioannis Papakonstantinou ${ }^{1}$, David R. Selviah ${ }^{1}$, Kai Wang ${ }^{1}$, \\ Richard A. Pitwon ${ }^{2}$, Ken Hopkins ${ }^{2}$, Dave Milward ${ }^{2}$ \\ ${ }^{1}$ Department of Electronic \& Electrical Engineering, University College London, Torrington Place, WC1E 7JE, London, \\ U.K \\ Email: d.selviah@ee.ucl.ac.uk \\ ${ }^{2}$ Xyratex, Langstone Road, Havant, Hants, PO9 1SA, U.K.
}

\begin{abstract}
The paper introduces a low cost, precision alignment technique designed to be unaffected by temperature or process variations in the thickness of the PCB FR4 board, the thickness of the lower cladding between the PCB board and the waveguide core, the thickness of the upper cladding above the waveguide core, the relative lateral position of waveguides across the PCB, and the axial position of the cut entrance and exit faces of the waveguide.
\end{abstract}

\section{Introduction}

Polymer multimode waveguides on or in printed circuit boards can provide low cost, high bit rate communications overcoming the cross-talk, loss, and distortion inherent in copper tracks [1]. To minimize the cost, optical connectors must align passively and precisely to the waveguides, have a low part count, use few processing steps and provide multiple duplex, communication channels in a compact unit. Passively self-aligning, MT-sockets give high alignment precision without the need for skillful assembly.

One of the key obstacles to the proliferation of optical backplane technology to date, has been the development of a viable method of dynamically registering arrays of active components on a mezzanine card to the waveguide interface of an optical printed circuit board (OPCB), while not compromising the critical optical alignment tolerances inherent to such arrangements. Most of the available connector designs so far are either restricted to optical fibers being aligned to the OPCB waveguides [2] [3] or to permanently flip-chipping VCSEL and PIN diodes onto the OPCBs [4] [5] preventing the connectors from being detachable. Limited work has been reported so far on connectors where active optical elements can be repeatedly plugged in and out from an OPCB with high precision [6] as in ordinary electronic PCBs.

This paper focuses on the design of a connector mechanism and its application to a novel OPCB connection scheme. The following method is proposed of coupling optical signals between an external photonic device and the polymeric optical waveguides of an OPCB. The active optical transmit and receive elements are drawn into physical alignment with the waveguide at a point of interface to the optical PCB, such that light can be launched directly into or out of the waveguides. This butt-coupling scheme eliminates the need for intermediary deflection optics on the backplane e.g. $45^{\circ}$ micro-mirrors [2] [5], and thus the additional cost and complexity of additional fabrication steps with precision assembly and alignment.

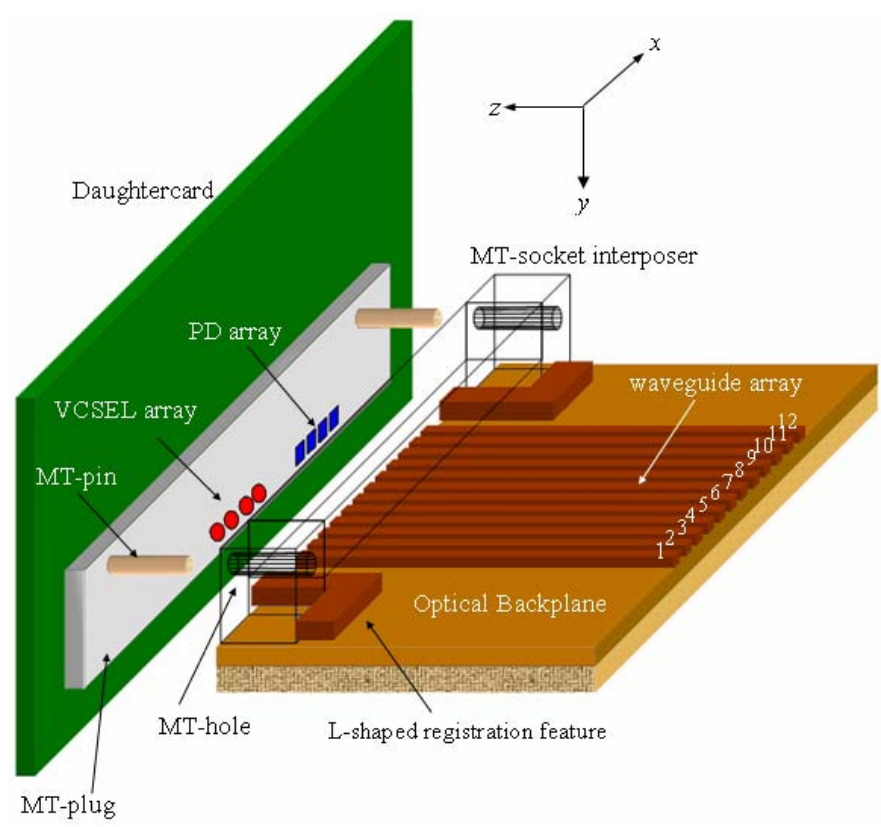

Figure 1: Passive aalignment method based on the MT-socket interposer / MT-plug concept.

\section{Passive Alignment Method}

Our passive alignment method relies on an MT-plug / MT-socket interposer mating interface, Figure 1, 2. The MTsocket interposer, Figure 1, Figure 2 (b), is an inverted Ushaped injection molded polymer structure that mechanically aligns with respect to the waveguide array with the aid of registration features fabricated in the core layer. The feet of the MT-socket interposer stand on the top of the lower cladding layer, which also acts as the FR4 planarizing layer. Since waveguides are also formed on the top of the same cladding layer, the alignment of the MT-socket interposer in the lateral $y$-direction is independent of any lower layers. The L-shaped mechanical registration features are fabricated by core material with the same e-beam mask and at the same step used to fabricate the waveguides. The sidewalls of the registration features, which run parallel to the waveguides, ensure precision alignment of the MT-socket interposer in the $x$-direction due to the high positional accuracy of the e-beam mask layout. Finally, the parts of the registration features that run vertically to the waveguides act as back stops which guarantee alignment in the axial $z$ - direction. Therefore, once the MT-socket interposer has been slotted into place laterally and slid back into contact with the backstops, alignment in 


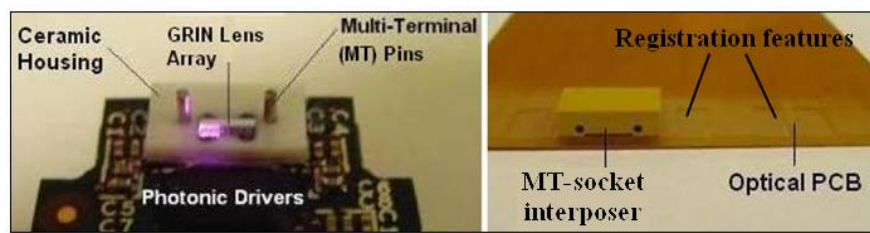

Figure 2: (a) MT-plug with ceramic housing in place. (b) $O P C B$ with MT-socket interposer mounted on its surface.

all three Cartesian directions is achieved between the waveguide array and the holes of the MT-socket interposer. Angular errors are expected to be negligible [7]. All active devices are mounted into the MT-plug, Figure 2 (a), Figure 3, leaving the backplane completely passive. A gradient index (GRIN) microlens array is positioned over the active emission and reception areas of the VCSEL and photodiode arrays respectively, with appropriate spacers to maintain the required free-space displacement between the microlenses and active emission and reception areas. The GRIN microlenses were designed to image the output from the VCSEL onto the polymeric waveguide surface and to image the output of the waveguide onto the active reception area of the PIN photodiode. The GRIN lens array and support structure served both to protect the fragile die underneath and to compensate for optical divergence from the VCSELs and the waveguides. In addition, the flat ingress / egress surfaces of the GRIN lenses made them suitable for butt coupling to the waveguide surfaces. The MT-plug contained MT-compatible alignment pin structures to engage with the compliant MTsocket interposer on the OPCB, Figure 1, 2, and draw the image points of the GRIN lens array into alignment with the waveguides.

\section{MT-plug}

As has already been mentioned the MT-plug carried all active devices. These included an array of four $10 \mathrm{~Gb} / \mathrm{s} 850 \mathrm{~nm}$ VCSELs with $7 \mu \mathrm{m}$ active diameters from ULM photonics [8] and an array of four $10 \mathrm{~Gb} / \mathrm{s}$ GaAs PIN diodes with $75 \mu \mathrm{m}$ active diameters [9] from Microsemi corporation. VCSELs and PDs were arranged on a pitch of $250 \mu \mathrm{m}$, which matched the waveguide to waveguide spacing on the OPCB. VCSELPD arrays were aligned and glued onto the MT-plug header using its raised alignment features under a microscope. The VCSELs were arranged to launch into waveguides 1-4 and PDs to receive from waveguides 9-12 of the OPCB in Figure 1. Waveguides 5-8 were unused in this first approach although they can be used in future applications. In this way, an 8-channel, $10 \mathrm{~Gb} / \mathrm{s} /$ channel bidirectional communication between two mezzanine cards was implemented.

The GRIN lenses were mounted on silicon V-grooves in a ceramic holder, Figure 3. The ceramic lens holder had two holes corresponding to the diameter of the MT-plug pins. So, when the MT-pins were slipped through the holes the VCSEL and PD images were brought into focus at the centre of the front surface of the GRIN lenses. A parallel optical transceiver was attached electrically to the line-card that hosted the MT-plug through a high-speed array connector, Figure 4. The active transmit and receive elements - VCSEL die array and PIN photodiode die array - were bonded

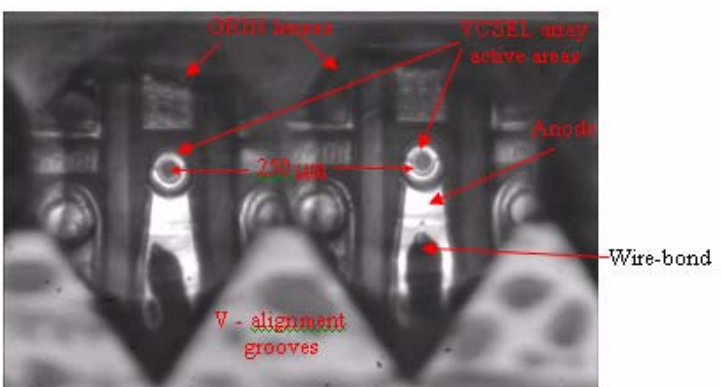

Figure 3: An array of GRIN-lenses held on V-alignment grooves aligned to the array of VCSELS (2 out of the 4 GRINlenses and VCSELs in the array are shown) was used to protect the wire bonds. Lenses were designed to image VCSEL output to the exterior surface of the lenses.

directly to the transceiver PCB. The drivers are the most power consuming elements in the transceiver circuit and in order to mitigate the risk of thermal overloading, mechanical holes were machined into the PCB under their location and copper structures were positioned in these holes such as to be in full thermal contact with the bases of the die. The copper structures guided the heat dissipated from the drivers into a heat sink positioned on the other side of the PCB, Figure 4.

The optical transceiver circuit was constructed on flexible laminate PCB with the sections housing the photonic interface and the mezzanine card connector supplemented with rigid FR4 layers. The FR4 was removed from the intermediary bridge between these sections such that it was mechanically flexible. This arrangement ensured that the photonic interface was free-floating with respect to the line-card, allowing the photonic interface to be manipulated into a precise alignment with the optical waveguides irrespective of the relatively coarse alignment of the line-card. The engagement mechanism, Figure 5, served two key functions:

1. Retraction of the photonic interface to protect the salient MT structures during the line card insertion and retraction process

2. Elevation of the photonic interface into engagement with compliant receptacle on the OPCB.

All precision assembly on the design is thus restricted to the photonic interface and the compliant receptacle.

\section{OPCB and MT-socket interposer}

The MT-socket interposer, Figure 2 (b), was machined from a block of Torlon ${ }^{\circledR}$ Polyamide-imide, with a thermal expansion coefficient chosen to be compatible with that of the FR4 to minimize thermal stress and $\mathrm{PCB}$ warping.

Each waveguide on the OPCB had a $70 \mu \mathrm{m} \times 70 \mu \mathrm{m}$ square cross section and a pitch of $250 \mu \mathrm{m}$. Waveguides were designed to be $10 \mathrm{~cm}$ long in order to fit on a 7" $\times 7$ " mask. A dark field e-beam mask was used for the fabrication of the optical features in the core, while a light field laser written mask was used for the deposition of the upper cladding layer. The fabrication of the OPCB took place in three distinct phases, Figure 6. In phase A, the $\sim 50 \mu \mathrm{m}$ thick lower cladding layer was deposited by spinning and UV curing. In phase $\mathrm{B}$, the core layer was spun on to a thickness of $70 \mu \mathrm{m}$ and waveguides and registration features were formed 


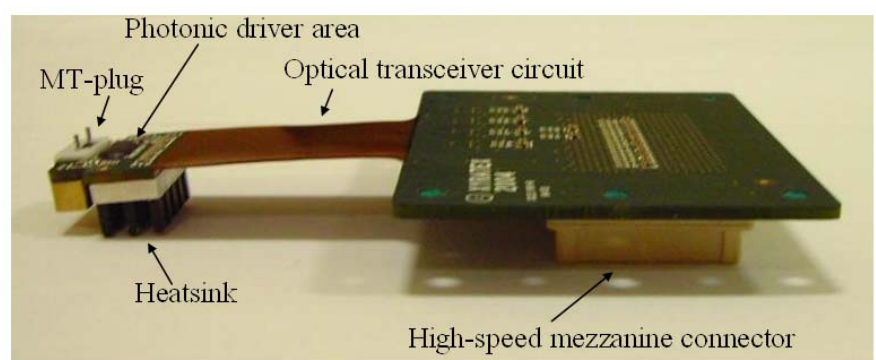

Figure 4: Line-card integrating high-speed electrical connector, optical transceiver circuits, photonic drivers, MTplug and VCSEL heatsink.

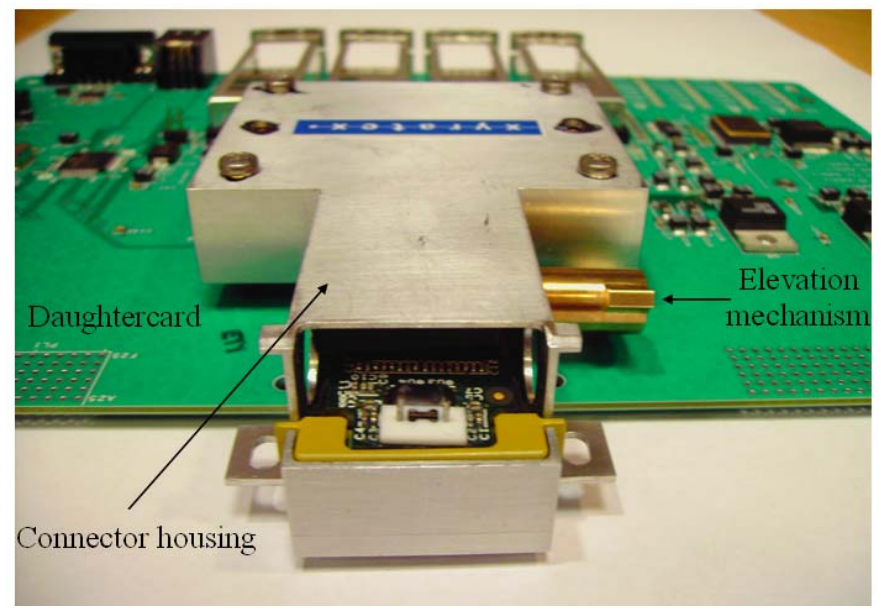

Figure 5: Line-card plugged onto a mezzanine card and metallic connector housing.

simultaneously photolithographically from the e-beam dark field mask. In phase $\mathrm{C}, \mathrm{a} \sim 50 \mu \mathrm{m}$ layer of upper cladding polymer was initially spun over the whole substrate. A second light field photomask, was then aligned visually to its cross alignment features left in the core layer from the first mask and the upper cladding was formed by UV illumination through the second mask and subsequent development clearing the unexposed residual material. The purpose of using a second mask was to prevent the upper cladding layer from covering the area adjacent to the registration feet, which had to remain clear at the end of the process for the feet of the MT-socket interposer to rest on the top of the lower cladding, Figure 6. The refractive index of the core was $n_{\text {core }}=1.556$ and that of the cladding was $n_{\text {clad }}=1.5264$, giving a waveguide numerical aperture of $N A=0.302$.

The tolerance of the MT-socket interposer to the waveguide alignment depends mainly on the accuracy with which the MT holes can be drilled and the over- or under-exposure that the polymer registration features experience upon UV illumination through the e-beam mask. In a well-calibrated mask aligner system the maximum tolerances of our alignment method were calculated to be $\pm 3 \mu \mathrm{m}$ in the $x$ direction, $\pm 4 \mu \mathrm{m}$ in the $y$-direction and $+10 \mu \mathrm{m}$ in the $z$ direction.
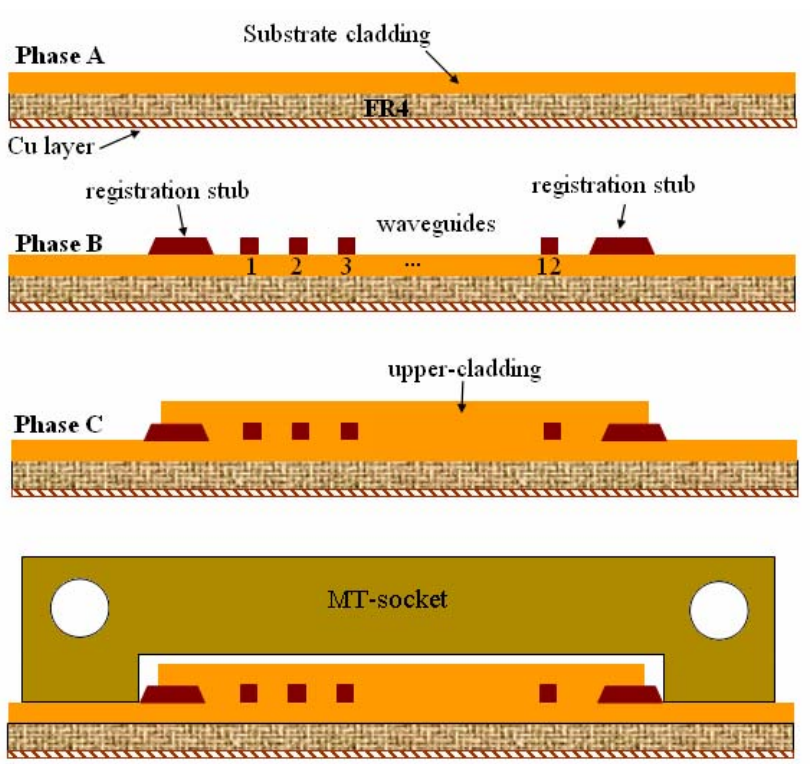

Figure 6: Schematic diagram of optical backplane fabrication. In Phase A lower substrate cladding was formed on the top of an 7" $\times 7$ " FR4 wafer. In phase B waveguides, registration stubs and back stops were formed simultaneously while in Phase $C$ upper-cladding was specially deposited to leave the area next to the registration stubs unfilled.

\section{VCSEL to Waveguide Coupling}

One important aspect of an optical link is how much of the light emitted by the laser source is coupled into the waveguides. The coupling efficiency is a parameter, which is not constant with the bias current $I_{\text {bias }}$ but it depends on the driving conditions of the VCSELs. Figure 7 (a) shows the power received at the end of one of the waveguides in the wafer as a function of $I_{\text {bias }}$, together with the power emitted from the VCSEL. The threshold current of the VCSELs was $I_{t h}=1 \mathrm{~mA}$. Measurements were calibrated to exclude propagation loss through the waveguides. On the same graph, we have plotted the coupling loss, which is defined as the difference (in $\mathrm{dB}$ ) between the VCSEL and waveguide powers. Since propagation loss has been calibrated out, coupling loss is the combination of Fresnel reflection losses and modal mismatch loss. No index matching fluid was used in these experiments. Figure 7 (a) reveals that as $I_{\text {bias }}$ increases, the coupling loss increases as well. In order to investigate further the relationship between the VCSEL emitted field and the coupling efficiency we measured the VCSEL far-field intensity distribution with a CCD camera and plotted the far-field divergence, defined as the full-width at $\mathrm{e}^{-2}$, and the coupling efficiency against $I_{\text {bias }}$, Figure 7 (b). Figure 7 (b) shows a strong correlation between the farfield divergence and the coupling efficiency. As $I_{\text {bias }}$ increases the far-field divergence increases while the coupling efficiency drops, even though in all cases the light emitted by the VCSEL was well within the waveguide $N A=0.302$ which corresponds to $\sim 34^{\circ}$ of full acceptance angle. As an example, at currents just above threshold, $I_{\text {bias }}=1 \mathrm{~mA}$ (threshold current was $0.7 \mathrm{~mA}$ ), coupling loss took its minimum value of $2.86 \mathrm{~dB}$, or $51.8 \%$ of light was coupled into the waveguide, 

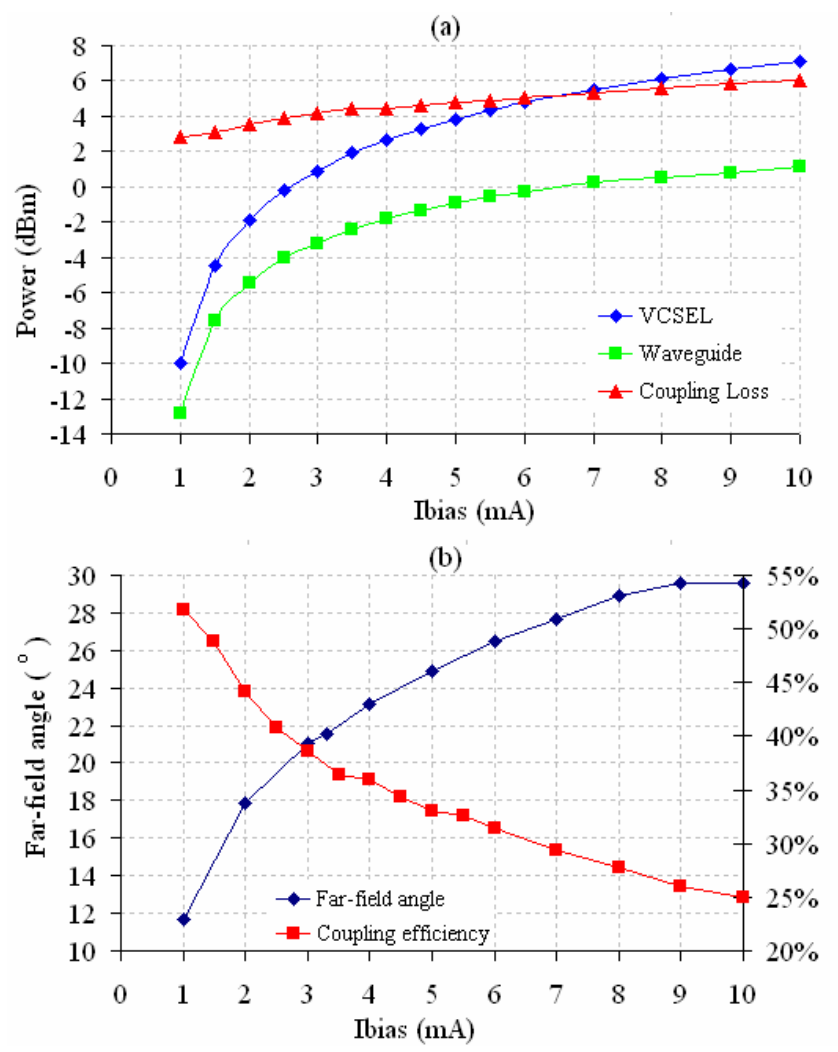

Figure 7: (a) VCSEL power, power at the output of the waveguide and insertion loss as a function of $I_{\text {bias }}$ (b) Farfield and coupling efficiency as a function of $I_{\text {bias. }}$.

while the far-field divergence was $\sim 11^{\circ}$. At $I_{\text {bias }}=10 \mathrm{~mA}$ the insertion loss increased to $6.031 \mathrm{~dB}$ or only $24.9 \%$ of light coupled into the waveguide, while the far-field divergence increased to $\sim 29^{\circ}$. In a binary optical system, a modulation (or pulse) current is superimposed onto the bias

current and $1 \mathrm{~s}$ and $0 \mathrm{~s}$ are represented by different levels of optical power. Therefore, for given bias and pulse currents, Figure 7 (a) can be utilized in optical link power budgets calculations.

Similar coupling behavior has been observed in [10], between VCSELs and multimode fibers. There it was shown that in multi-transverse mode emitting VCSELs, as the ones we are using, the coupling efficiency strongly depended on the VCSEL transverse modes. The coupling efficiency was better for low $I_{\text {bias }}$ where VCSELs exhibited nearly single mode operation but it degraded for higher $I_{\text {bias }}$ where high order lasing modes made their appearance and as a result, the far-field divergence of the VCSEL increased. The higher transverse modes of the VCSELs were more spatially dissimilar to the fiber modes than the low order modes and therefore they could not be coupled with the same efficiency. The form of Figure 7 (b) suggests that this may occur in our case.
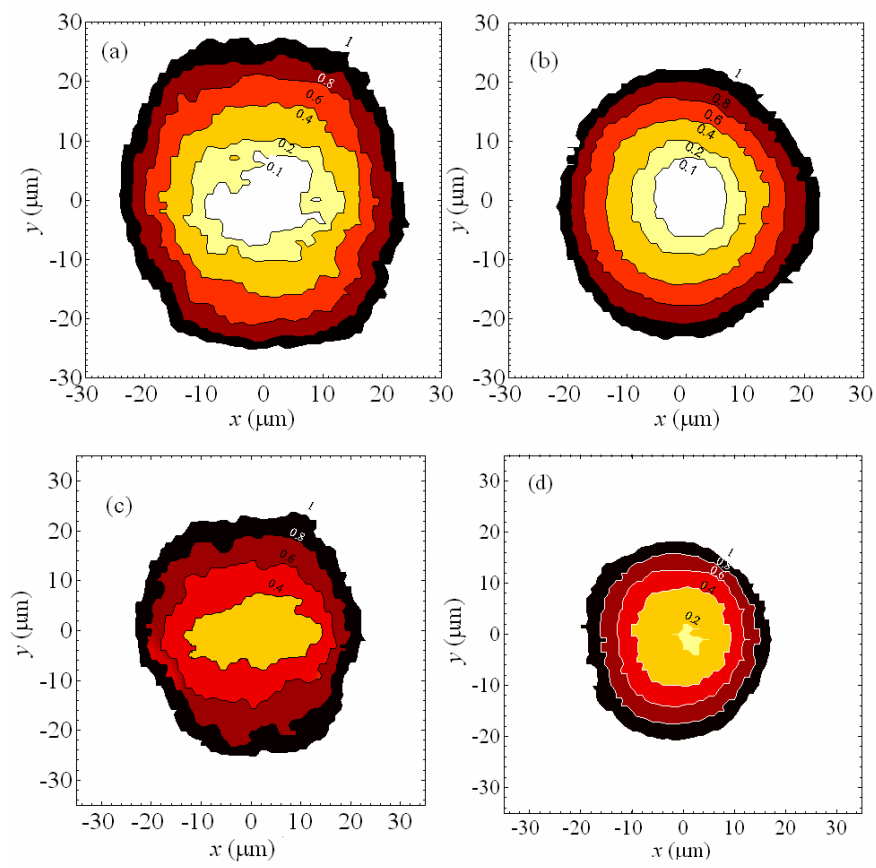

Figure 8: Contour map of relative insertion loss compared to the maximum coupling position for VCSEL lateral misalignments at $z=0$. (b) Same for lateral $P D$ misalignments at $z=0$. (c) Contour map of relative insertion loss for VCSEL lateral misalignments at $z=60 \mu \mathrm{m}$. (d) Same for PD misalignments at $\Delta z=60 \mu \mathrm{m}$. Resolution step was $\Delta x$ $=\Delta y=1 \mu \mathrm{m}$. Waveguide size $70 \mu \mathrm{m} \times 70 \mu \mathrm{m}$.

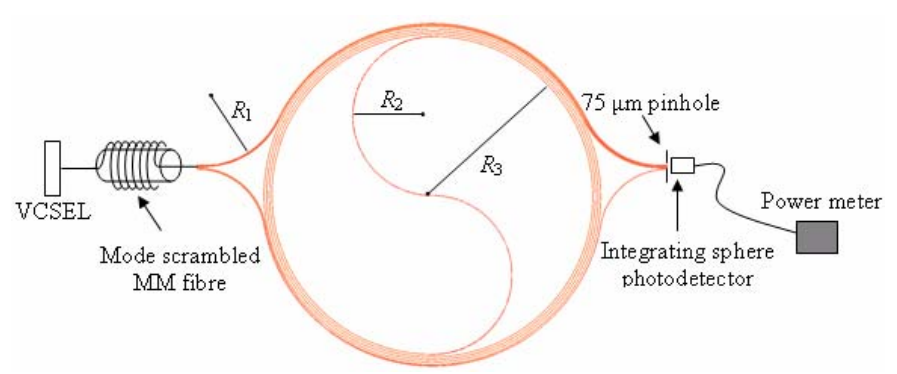

Figure 9: A 1 m-spiral waveguide used to measure propagation loss. VCSEL light was coupled to the spiral waveguide through a MM mode scrambled fiber. A $75 \mu \mathrm{m}$ pinhole at the waveguide output spatially filtered cladding light out allowing an integrating sphere photodetector to collect light from the waveguide core only. Index matching fluid was used in this set of experiments. 


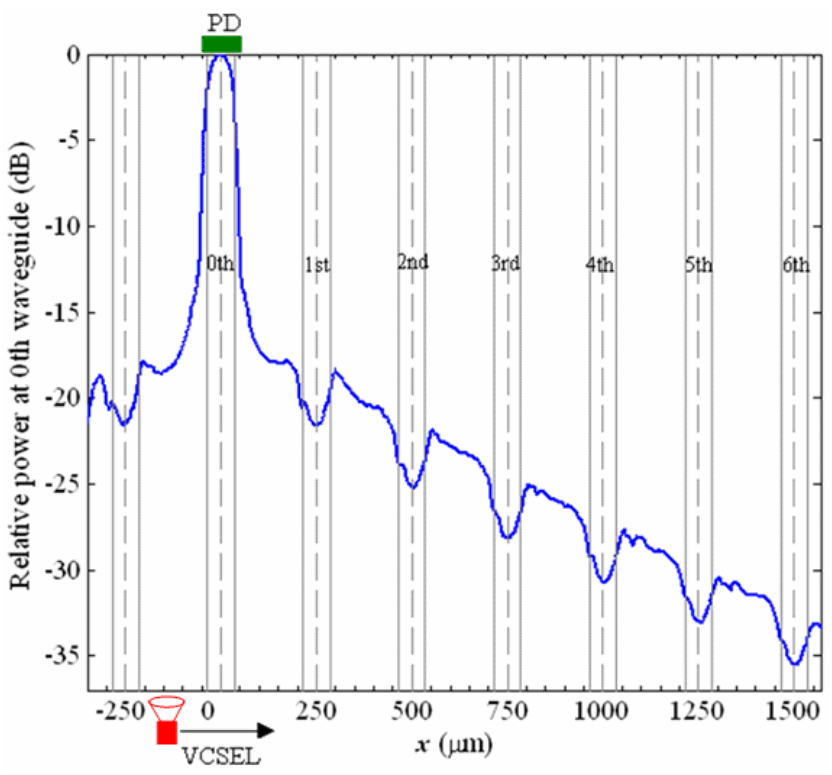

Figure 10: Power received at the end of 0th waveguide as a function of the lateral distance of the VCSEL. The resolution of the scans was $1 \mu \mathrm{m}$. Figure after [12].

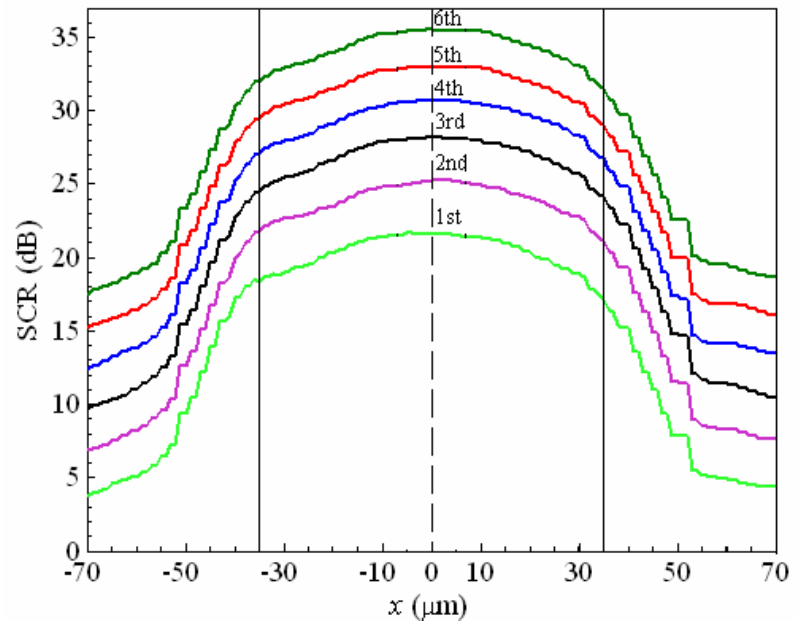

Figure 11: (a) Signal-to-cross-talk (SCR) levels that 0th waveguide experiences from its adjacent waveguides. Figure after [12].

\section{Insertion Loss due to Active Component Misalignment}

In order to measure the insertion loss as a function of the three possible translational misalignments in our prototype connector design we positioned an MT-plug with its pins being removed on a system of three $x y z$-motorized stages and brought

the GRIN lens array in contact with the waveguides on the wafer. We first measured the waveguide that was butted against the VCSEL and scanned the MT-plug along $x$, and $y$ at different positions of $z$, while monitoring the power at the waveguide output. In this way we generated $x y$-contour maps of insertion loss due to VCSEL misalignment, Figure 8 (a) for $z=0 \mu \mathrm{m}$ and (c) for $z=60 \mu \mathrm{m}$. Similarly, by measuring the waveguide that was butted against a PD and fixing the VCSEL at the other end of the wafer at the best coupling position we generated $x y$-contour maps of insertion loss due to PD misalignment by scanning the MT-plug, Figure 8 (b) for $z=0 \mu \mathrm{m}$ and (d) for $z=60 \mu \mathrm{m}$. The VCSEL bias current was set to $I_{\text {bias }}=3.3 \mathrm{~mA}$, DC, which provided an output optical power of $0 \mathrm{dBm}$. The position of minimum insertion loss corresponded to $x=y=z=0$ on the contour maps. The insertion loss showed in the contour maps of Figure 8 is relative to the minimum insertion loss measured. Note that no index matching fluid was used in these experiments. The $z=0$ $\mu \mathrm{m}$ case corresponded to the VCSELs or PDs being buttcoupled to the waveguides. The $z=60 \mu \mathrm{m}$ case, is just an example to illustrate the effect of $z$ displacement on the contour maps.

The contour maps in Figure 8 indicate the accuracy that the position of either the VCSEL or the PD should be controlled in order to achieve a targeted insertion loss. Therefore, from these graphs we can extract the requirements for a connector design. As we see for $z=0 \mu \mathrm{m}$, the VCSELs or PDs can be positioned in an area with a radius of more than $\pm 25 \mu \mathrm{m}$ on the $x y$-plane before the received power drops by 1 $\mathrm{dB}$. The effect of an increased misalignment $z$ is the shrinking of the contour maps, for the same levels of insertion loss.

\section{Insertion and Propagation Losses}

The insertion loss for the waveguides in our system was measured in the range $4.4 \mathrm{~dB}-4.8 \mathrm{~dB}$, without index matching fluid, depending on the waveguide under investigation. Applying index index-matching fluid improved the results by $2.1 \mathrm{~dB}-2.7 \mathrm{~dB}$, which is attributed to the combined effect of the mode mismatch loss, any remaining Fresnel reflections, and any remaining scattering from the waveguide entrance and exit facets. We note that the waveguides were diced but not polished in a low cost approach and they were quite rough with a $250 \mathrm{~nm}$ RMS roughness. Therefore, coupling loss was expected to be quite high in the case without index matching fluid.

The propagation loss due to material absorption and scattering from the sidewalls was calculated with the aid of three $1 \mathrm{~m}$ spiral waveguides made under the same conditions with our wafer, Figure 9. The radii of the waveguide bends in the spiral were designed to minimize bend loss according to [11]. The average propagation loss from the three spirals was measured to be $\alpha=0.08 \pm 0.01 \mathrm{~dB} / \mathrm{cm}$. Therefore, our $10 \mathrm{~cm}$ long waveguides exhibited $0.8 \mathrm{~dB}$ of propagation loss in total. If we remove the mode mismatch loss, the Fresnel reflections, the scattering and the propagation loss from the insertion loss, then the remaining $0.9-1.9 \mathrm{~dB}$ is the coupling loss between the VCSELs and the waveguides and between the waveguide and the PDs. Therefore, sub-1 dB of coupling loss, typical in MT-fiber ribbon connectors [7], is feasible with our connector design in a real system if the waveguides are polished and adequate contact force is applied between the microlens array and the waveguides.

\section{Waveguide-to-Waveguide Cross-talk}

The performance of high-bit rate systems is directly affected by cross talk. In order to measure the cross talk in our system, we positioned a VCSEL at the best coupling position, $y=z=0$, in front of the $0^{\text {th }}$ waveguide, Figure 10 , and scanned it laterally in the $x$-direction. Our scan covered the area from one waveguide to the left to six waveguides to the right of the $0^{\text {th }}$, while the power at the output of the $0^{\text {th }}$ 
(a)

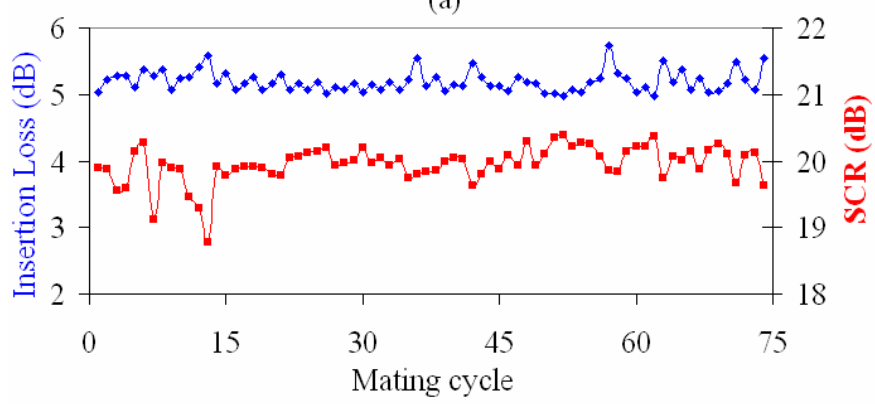

(b)

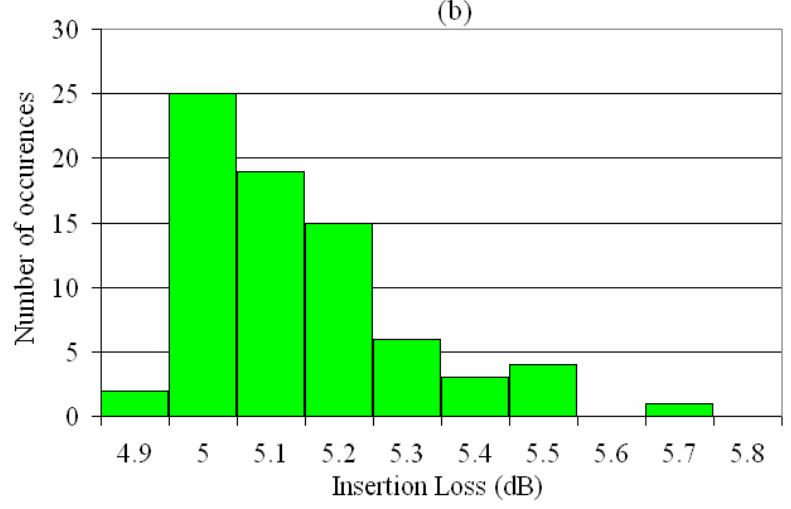

Figure 12: (a) Insertion loss and signal to cross talk (SCR) as a function of mating cycle for 75 engagements. (b) Histogram of insertion loss. Figure after [12].

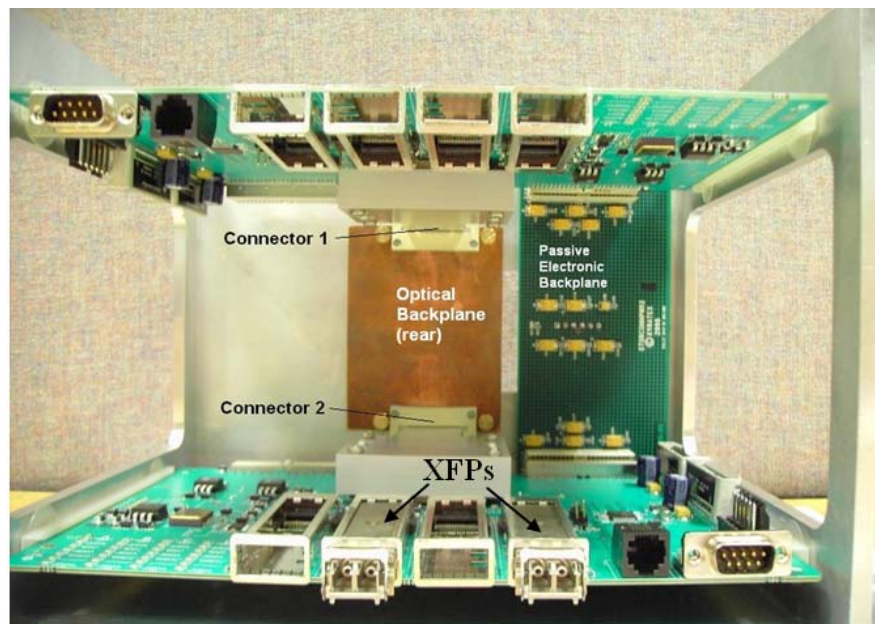

Figure 13: Optical backplane prototype with two mezzanine cards being interconnected.

waveguide was recorded at each step. When the VCSEL was moving within the core of the $0^{\text {th }}$ waveguide, the received power corresponded to the power coupled into this waveguide as a function of $x$. However, when the VCSEL was scanning the cores of the neighboring waveguides, the detected power corresponded to the cross talk, which would be due to the other VCSELs in the array if they had been turned on. Figure 10, [12] shows that the power received in the waveguide under investigation reduced almost linearly at a rate of 0.011 $\mathrm{dB} / \mu \mathrm{m}$, as the VCSEL moved in the cladding away from the waveguide. However, sudden drops in the power received were observed when the VCSEL reached each of the adjacent waveguides, due to power being trapped within the waveguides' cores.

The four VCSELs mounted in the MT-plug were part of an array and so have the same amount and direction of misalignment, if no rotational misalignment is present. The signal to cross-talk ratio (SCR) between any two waveguides for a specific amount of misalignment $x$ can then be calculated from Figure 10. This is done by subtracting (in $\mathrm{dBs}$ ) the power coupled into the $0^{\text {th }}$ waveguide when the VCSEL is $x \mu \mathrm{m}$ away from its center, from the power detected when the VCSEL is moved to the same position relative to the centre in the neighboring waveguide. According to this assertion, the lowest cross talk levels are achieved when perfect alignment occurs between VCSELs and waveguides as can be seen from Figure 11. The crosstalk between two adjacent waveguides separated by $250 \mu \mathrm{m}$ is $-21.5 \mathrm{~dB}$ when no misalignment occurs, which drops to -25 $\mathrm{dB}$ for waveguides $500 \mu \mathrm{m}$ apart and to $-28.5 \mathrm{~dB}$ for waveguides $750 \mu \mathrm{m}$ apart, which is the maximum separation between waveguides used in our prototype system. As the VCSELs move away from perfect alignment, the cross-talk becomes worse.

\section{Connector Repeatability}

The insertion loss and the cross talk were measured for one of the channels in our prototype connector, as we engaged the MT-socket into the MT-plug 75 times, Figure 12, [12]. To measure the insertion loss, only the VCSEL of the channel under consideration was switched on while the other three VCSELs in the array were switched off.
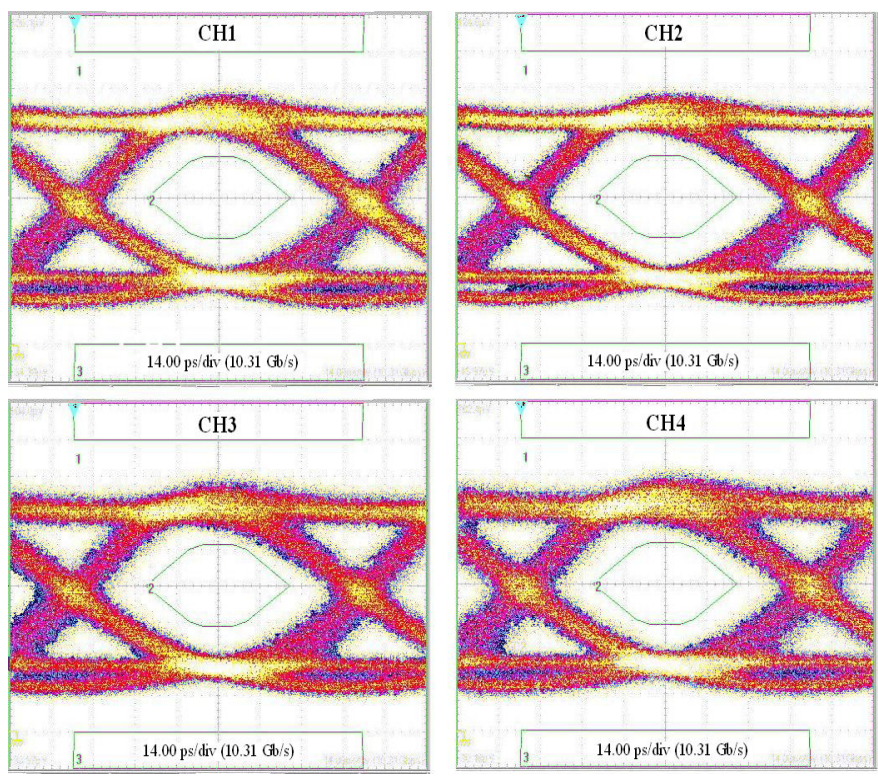

Figure 14: $10.3 \mathrm{~Gb} / \mathrm{s}$ eye diagrams for four channels in our prototype system demonstrator.

The average insertion loss measured was $5.19 \pm 0.16 \mathrm{~dB}$, with the histogram of insertion loss shown in Figure 12 (b) having the usual form of a one-sided Gaussian for well-aligned connectors. The standard deviation of the insertion loss 
achieved by our connector, $0.16 \mathrm{~dB}$, is comparable to that of single mode connector systems.

To measure the combined cross-talk from the other three channels, we switched the VCSEL of the channel under investigation off and switched the rest three VCSELs on. The average received cross talk was $-19.95 \pm 0.27 \mathrm{~dB}$ in agreement with the expected values from Figure 11 .

\section{Test Line Card Functionality}

The integrated transceiver devices were supported on a test card, which served as a physical layer bridge for $10 \mathrm{GbE}$ LAN traffic between the transceiver and an external test traffic source. In order to provide fully exercise the quad duplex channels of the transceiver prototype, 4 commercial XFP optical transceivers were housed on the front edge of the line card, each XFP comprising a single high speed transmit and receive channel and provided clock and data recovery on each signal path, Figure 13. $10 \mathrm{GbE}$ LAN test traffic was delivered optically through a fiber connection to the XFPs. The optical signal was then converted into an electronic signal by the XFP circuit and launched onto the line card. The linecard was composed of a low-loss dielectric Rogers material, which minimized signal distortion along the electrical signal traces. The high speed signals were conveyed electrically to the prototype transceiver through the high-speed array connector on the transceiver and launched into the OPCB as described in the previous section. Likewise, optical signals received from the OPCB are delivered onto the line card across the array connector, conveyed to the XFP modules, and reconverted to an optical signal, which is then sent across an optical fiber connection to a $10 \mathrm{GbE}$ LAN test traffic analyzer. A PIC microcontroller was used to regulate the XFPs and the supported transceiver.

Typical eye-diagrams obtained for four channels in our prototype are shown in Figure 14. Eye-diagrams were clean with measured jitter $<35$ ps (or less $35 \%$ of the bit dutycycle), in all cases. Error-free operation was observed after passing 10G Ethernet frames through our prototype OPCB for more than $48 \mathrm{~h}$ qualifying the system for Ethernet protocol based applications.

\section{Conclusions}

A prototype demonstrator in which two mezzanine cards were interconnected via an OPCB was presented. Eight 10 $\mathrm{Gb} / \mathrm{s}$ bidirectional channels provided an aggregate $80 \mathrm{~Gb} / \mathrm{s}$ bandwidth to the cards. A novel low cost connector design for passive butt coupling between arrays of active components on the mezzanine cards and the backplane waveguides with high accuracy and low insertion loss was implemented in our prototype design. This research brings forward the day when OPCBs replace $\mathrm{PCBs}$.

\section{Acknowledgement}

This research was supported by EPSRC via the Storlite and the IeMRC OPCB projects. We thank Dr. Navin Suyal, Exxelis Ltd. for fabricating the waveguides and Dr. Frank Tooley, Photonix Ltd. for useful discussions.

\section{References}

1. D. A. B. Miller, "Rationale and Challenges for Optical Interconnects to Electronic Chips", Proc. IEEE, vol. 88, pp. $728-749$, Jun. 2000

2. G. V. Steenberge, P. Geerinck, S. V. Put, J. V. Koetsem, D. Morlion, P. V. Daele, "Demonstration of an MTcompatible connectorisation of a laser-ablated optical interconnection on a printed circuit board," Proc $54^{\text {th }}$ Electronic Components and Technology Conference, 2004

3. A. L. Glebov, M. G. Lee, and K. Yokouchi, "Integration technologies for pluggable backplane optical interconnect systems," Opt. Eng., vol. 46, pp. 015403 1-10, Jan. 2007.

4. S. Hiramatsu, and T. Mikawa, "Optical design of active interposer for high-speed chip level optical interconnects," J. Lightw. Technol., vol. 24, pp. 927-934, Sep. 2006

5. B. S. Rho, H. S. Cho, H.-H. Park, S.-W. Ha, and B.-H. Rhee, "PCB-compatible optical interconnection using $45^{\circ}$-ended connection rods and via-holed waveguides," $J$. Lightw. Technol., vol. 22, pp. 2128-2134, Sep. 2004

6. D L. Dellmann, C. Berger, R. Beyeler, R. Dangel, M. Gmur, R. Hamelin, F. Horst, T. Lamprecht, N. Meier, T. Morf, S. Oggioni, Mauro Spreafico, R. Stevens, B. J. Offrein, "120 Gb/s Optical Card-to-Card Interconnect Link Demonstrator with Embedded Waveguides," Proc $57^{\text {th }}$ Electronic Components and Technology Conference, 2007

7. Masaaki Takaya, and Koji Shibata, "Design and performance of very-high-density 60-fiber connectors," J. Lightw. Technol., vol. 21, pp. 1549-1556, Jun. 2003

8. ULM photonics, $10 \mathrm{~Gb} / \mathrm{s}$ VCSEL array data-sheet. Available at http://www.ulm-photonics.de/docs/products _new/datasheets/VCSEL-ULM850-10-chips.pdf. Last time accessed 16/06/2007

9. Microsemi corporation, LX3045 $10 \mathrm{~Gb} / \mathrm{s}$ coplanar GaAs PIN photodiode data-sheet. Available at http://www.microsemi.com/datasheets/lx304x.pdf. Last time accessed 16/06/2007

10. J. Heinrich, E. Zeeb, and K. J. Ebeling, "Butt-Coupling Efficiency of VCSEL's into Multimode Fibres," IEEE Photon. Technol. Lett., vol. 9, pp. 1555-1557, Dec. 1997

11. I. Papakonstantinou, K. Wang, D. R. Selviah., and F. A. Fernández, "Transition, radiation and propagation loss in polymer multimode waveguide bends," Opt. Express, vol. 15, pp. 669-679, Jan. 2007

12. I. Papakonstantinou, D. R. Selviah, R. A. Pitwon, and D. Milward, "Low cost, precision, self-alignment technique for coupling laser and photodiode arrays to waveguide arrays." Accepted for publication, IEEE Transactions on Advanced Packaging, 2008 